Appl. Set-Valued Anal. Optim. 1 (2019), No. 1, pp. 77-94

Available online at http://asvao.biemdas.com

https://doi.org/10.23952/asvao.1.2019.1.07

\title{
SET OPTIMIZATION PROBLEMS ON ORDERED SETS
}

\author{
JINLU LI ${ }^{1}$, CHRISTIANE TAMMER ${ }^{2, *}$ \\ ${ }^{1}$ Department of Mathematics, Shawnee State University, Portsmouth, Ohio 45662, USA \\ ${ }^{2}$ Institute of Mathematics, Martin-Luther-University Halle-Wittenberg, Theodor-Lieser Str. 5, 06120 Halle, Germany
}

\begin{abstract}
In this paper, we consider optimization problems of set valued mappings on ordered sets. It leads us to define or to construct some ordering relations on the power sets of ordered sets, which play the standards for ordering different sets. On the power sets of preordered sets, we introduce three preordering relations based on the given preorder relations. Several properties of these preordering relations are provided. Under these preordering relations, we prove some order-clustered fixed point theorems, which are applied to prove existence theorems of extended Nash equilibriums for set-valued mappings.
\end{abstract}

Keywords. Preordered set; Order-clustered fixed point; Power preorder; Extended Nash equilibrium.

2010 Mathematics Subject Classification. 47H10, 90C33.

\section{INTRODUCTION}

For some optimization problems in traditional optimization theory with given real valued function, one seeks a point or points in the domain at which the considered function will take the maximum value or the minimum value; see $[3,4,17,18]$ and the references therein. The traditional optimization theory has been extended to the cases that the ranges of the considered functions in optimization problems are not linearly ordered. For example, they may be preordered sets, partially ordered sets, or lattices. The considered that optimization problems become ordered optimization problems or vector optimization problems; see $[6,13,14,18]$ and the references therein. One more step further, if the considered mappings in optimization problems are set-valued mappings, then it raises the so-called set optimization problems; see $[5,7,8,9,10,11,12]$ and the references therein. In any optimization problem, in the ranges of considered mappings, there may not have any algebraic and topological structures. But there must be some ordering relations on the range of the considered mappings, that play the important roles for comparing the preferences of different outcomes. The most general, useful and realistic structures on the ranges are preorder relations. It is the motivation of this paper to study the set optimization problems on preordered sets.

In this paper, we study the very general set optimization problems of the following types. Suppose that the underlying space $Z$ is equipped with a preordering relation $\succcurlyeq$, so that $(Z, \succcurlyeq)$ becomes a preordered set (This concept is very general. It includes the special cases, such as, partially ordered set, lattice, partially ordered vector spaces, Banach lattices, etc.). Based on the preordering relation $\succcurlyeq$ on $Z$, we must

${ }^{*}$ Corresponding author.

E-mail addresses: jli@ shawnee.edu (J. Lu), christiane.tammer@ mathematik.uni-halle.de (C. Tammer).

Received February 26, 2019; Accepted April 5, 2019.

(C)2019 Applied Set-Valued Analysis and Optimization 
define (or construct) an ordering relation on the power set $2^{Z}$. Under this ordering relation on $2^{Z}$, we can introduce the comparison of the preferences of the subsets of $Z$. Then, for any set valued mapping $T: Z \rightarrow 2^{Z}$, we can introduce the concept of the optimization of $T$. For example, some authors defined an ordering relation $\gtrsim$ on $2^{Z}$ based on the preordering relation $\succcurlyeq$ on $Z$ as follows: for any $A, B \in 2^{Z}$,

$$
A \gtrsim B \text { if and only if } a \succcurlyeq b \text {, for every } a \in A \text { and for every } b \in B \text {. }
$$

We think that the ordering relation $\gtrsim$ on $2^{Z}$ as defined by (1.1) is too strong. It is because that to have $A$ is better than $B$, if and only if every member $a \in A$ must be better than every $b \in B$. This may not be reasonable in some applications in the real world. Hence, in this paper, we define three preordering relations on the power set $2^{Z}$ induced by $\succcurlyeq$ on $Z$, which are weaker than ordering relation $\gtrsim$ defined in (1.1) and are more useful in applications.

In Section 2, we present the properties of the order-cluster sets of preordered sets in general. Based on the preorders, we introduce three preorder relations on the power sets. Several properties of the three preorders are provided in this section. In the last subsection of the Section 2, we prove some existence theorems for order-clustered fixed points, which can be used in the future study for the solvability of some set optimization problems on preordered spaces. In Section 2, we introduce the concepts of extended Nash equilibrium and order-clustered extended Nash equilibrium for set-valued mappings. Then, by applying the order-clustered fixed point theorems proved in Section 2, we prove several existence theorems of extended Nash equilibrium and order-clustered extended Nash equilibrium for set-valued mappings.

\section{PREORdered SETS AND ORDER-Cluster SPACES}

2.1. Order-clusters and order-clustered fixed points in preordered sets. Let $(Z, \succcurlyeq)$ be a preordered set. For $z_{1}, z_{2} \in Z$, if neither $z_{2} \succcurlyeq z_{1}$, nor $z_{1} \succcurlyeq z_{2}$, then $z_{1}$ and $z_{2}$ are said to be not $\succcurlyeq$-comparable and it is denoted by $z_{1} \bowtie z_{2}$. If $z_{2} \succcurlyeq z_{1}$ and $z_{1} \succcurlyeq z_{2}$ both hold, then $z_{1}$ and $z_{2}$ are said to be $\succcurlyeq$-equivalent and it is denoted by $z_{1} \backsim z_{2}$. In this paper, we keep the same traditions in order theory about the ordering

relations. For example, $z_{2} \succcurlyeq z_{1}$ is equivalent to $z_{1} \preccurlyeq z_{2}$ and $z_{2} \succcurlyeq z_{1}$ means that $z_{2} \backsim z_{1}$ or $z_{2} \succ z_{1}$. For every $z \in Z$, we denote

$$
[z]_{\succcurlyeq}=[z]=\{t \in Z: t \sim z\} .
$$

The foot note $\succcurlyeq$ in $[z]_{\succcurlyeq}$ may be dropped and $[z]_{\succcurlyeq}$ is simply denoted by $[z]$ if there is no confusion caused. $[z]$ is called the $\succcurlyeq$-cluster in $Z$ containing $z$ (simply called the cluster in $Z$ containing $z$ if there is no confusion caused). It is clear to see that $\sim$ is an equivalent relation on $Z$ and the set of all clusters in $Z$ is written as

$$
[Z]_{\succcurlyeq}=[Z]=\{[z]: z \in Z\} .
$$

$[Z]$ is a partition of $Z$. [Z] is called the $\succcurlyeq$ cluster space (simply called the cluster space) of $Z$ with respect to the preorder $\succcurlyeq$. For any nonempty subset $A \subseteq Z,(A, \succcurlyeq)$ is also a preordered set. We denote

$$
[A]_{\succcurlyeq}=[A]=\{[z]: z \in A\} .
$$

For every $z \in Z$, the $\succcurlyeq$-cluster $[z]$ is an element of the $\succcurlyeq$-cluster space $[Z]$ of $Z$. On the other hand, as $[z]$ being considered be a subset of $Z$, we have

$$
z \in[z], \text { for every } z \in Z
$$


and

$$
A \subseteq \cup[A]=\cup_{z \in A}[z] \text {, for any } A \subseteq Z .
$$

Notice that, in a preordered set $(Z, \succcurlyeq)$, every cluster is a singleton if and only if $(Z, \succcurlyeq)$ is a partially ordered set (simply called a poset). If $(Z, \succcurlyeq)$ is a partially ordered set, then

$$
\{z\}=[z], \text { for every } z \in Z,
$$

and

$$
A=[A], \text { for every } A \subseteq Z .
$$

Next, we introduce the concept of order-clustered fixed points on preordered sets that is an extension of the concept of fixed points for single valued mappings on partially ordered sets; see [16] and the references therein.

Definition 2.1. Let $(Z, \succcurlyeq)$ be a preordered set and $D$ a nonempty subset of $Z$. Let $f: D \rightarrow D$ be a single valued mapping. A point $z \in D$ is called an $\succcurlyeq$-clustered fixed point (simply called a clustered fixed point) of $f$ if $z \in[f(z)]$.

It immediately follows that if $(Z, \succcurlyeq)$ is a poset, then a point $z \in D$ is an $\succcurlyeq$-clustered fixed point of a single-valued mapping $f$ if and only if $z$ is a fixed point of $f$.

Next definition is an extension of Definition 2.1 from single-valued mappings to set-valued mappings (multifunction).

Definition 2.2. Let $(Z, \succcurlyeq)$ be a preordered set and $D$ a nonempty subset of $Z$. Let $F: D \rightarrow 2^{D}$ be a set-valued mapping. $A$ point $z \in D$ is called an $\succcurlyeq$-clustered fixed point (simply called a clustered fixed point) of $F$ if $z \in[v]$, for some $v \in F(z)$. By (2.1), it is $[z] \in[F(z)]$.

Similar, to the remarks after Definition 2.1, if $(Z, \succcurlyeq)$ is a poset, then the set of $\succcurlyeq$-clustered fixed points of a set-valued mapping $F$ coincides with the set of fixed points of $F$.

Notations: Let $(Z, \succcurlyeq)$ be a preordered set. Let $F: Z \rightarrow 2^{Z}$ be a set-valued mapping. We denote $\mathfrak{F}(F, \succcurlyeq)$ (simply by $\mathfrak{F}(F)$ ) and $\mathscr{F}(F)$ for the set of $\succcurlyeq$-clustered fixed points and the set of fixed points of $F$, respectively. In general, we have

$$
\mathscr{F}(F) \subseteq \mathfrak{F}(F) .
$$

2.2. Ordering relations on power sets. Let $(Z, \succcurlyeq)$ be a preordered set. We define three preordering relations on $2^{Z}$ based on $\succcurlyeq$, that are called the power preorder, the upward power preorder and the downward power preorder relations, as follows.

(a) The power preorder, denoted by $\succcurlyeq^{P}$. For any $B_{1}, B_{2} \in 2^{Z}$, we say that $B_{1} \preccurlyeq^{P} B_{2}$ if the following two conditions are satisfied:

$(U)$ upward condition: for any $z_{1} \in B_{1}$, there is $t_{2} \in B_{2}$ such that $t_{2} \succcurlyeq z_{1}$;

$(D)$ downward condition: for any $z_{2} \in B_{2}$, there is $t_{1} \in B_{1}$ such that $t_{1} \preccurlyeq z_{2}$.

(b) The upward power preorder, denoted by $\succcurlyeq^{U}$. For any $B_{1}, B_{2} \in 2^{Z}$, we say that $B_{1} \preccurlyeq^{U} B_{2}$ if the upward condition (U) is satisfied.

(c) The downward power preorder, denoted by $\succcurlyeq^{D}$. For any $B_{1}, B_{2} \in 2^{Z}$, we say that $B_{1} \preccurlyeq^{D} B_{2}$ if the downward condition (D) is satisfied. 
We give some properties below about the three preordering relations on the power set.

Lemma 2.1. Let $(Z, \succcurlyeq)$ be a preordered set. Let $\succcurlyeq^{P}, \succcurlyeq^{U}$ and $\succcurlyeq^{D}$ be the power preorder, upward power preorder and downward power preorder, respectively, on the power set $2^{Z}$ induced by $\succcurlyeq$ on $Z$. We have

(i) $\succcurlyeq^{P}, \succcurlyeq^{U}$ and $\succcurlyeq^{D}$ all are preorders on $2^{Z}$. So, $\left(2^{Z}, \succcurlyeq^{P}\right),\left(2^{Z}, \succcurlyeq^{U}\right)$ and $\left(2^{Z}, \succcurlyeq^{D}\right)$ all are preorder sets;

(ii) For any $B \in 2^{Z} \backslash\{\emptyset\}, B$ and $\emptyset$ are not $\succcurlyeq^{P}$-comparable in $\left(2^{Z}, \succcurlyeq^{P}\right)$, that is,

$$
B \bowtie^{P} \emptyset, \text { for any } B \in 2^{z} \text { with } B \neq \emptyset \text {. }
$$

(iii) $\emptyset$ is the $\succcurlyeq^{U}$-least element and $Z$ is the $\succcurlyeq^{U}$-greatest element in $\left(2^{Z}, \succcurlyeq^{U}\right)$, that is,

$$
\emptyset \preccurlyeq^{U} B \preccurlyeq^{U} Z \text {, for any } B \in 2^{z} \text {. }
$$

(iv) $\emptyset$ is the $\succcurlyeq^{D}$-greatest element and $Z$ is the $\succcurlyeq^{D}$-least element in $\left(2^{Z}, \succcurlyeq^{D}\right)$, that is,

$$
Z \preccurlyeq^{D} B \preccurlyeq^{D} \emptyset \text {, for any } B \in 2^{z} \text {. }
$$

(v) $\succcurlyeq^{P}$ is "weaker" than both $\succcurlyeq^{U}$ and $\succcurlyeq^{D}$, that is,

$$
\text { for any } B_{1}, B_{2} \in 2^{Z}, B_{1} \preccurlyeq^{P} B_{2} \text { implies both } B_{1} \preccurlyeq^{U} B_{2} \text { and } B_{1} \preccurlyeq^{D} B_{2} \text {. }
$$

Proof. The proof of Lemma 2.1 is straightforward, and it is omitted here.

In this paper, we concentrate on preordered sets. One of the main reasons is the following.

Remark 2.1. No matter that $(Z, \succcurlyeq)$ is a preordered set or a partially ordered set, the ordering relations $\succcurlyeq^{P}$ ,$\succcurlyeq^{U}$ and $\succcurlyeq^{D}$ on $2^{Z}$ are always preorders. So, $\left(2^{Z}, \succcurlyeq^{P}\right),\left(2^{Z}, \succcurlyeq^{U}\right)$ and $\left(2^{Z}, \succcurlyeq^{D}\right)$ all are always preordered sets.

We provide some examples below about the three preorders on the power set, which are the extreme cases. It is stated as a lemma.

Lemma 2.2. Let $(Z, \succcurlyeq)$ be a preordered set as stated in Lemma 2.1. For any $B_{1}, B_{2} \in 2^{Z}$, we have

(a) If $B_{2}$ contains an $\succcurlyeq$-upper bound of $B_{1}$ and $B_{1}$ contains an $\succcurlyeq$-lower bound of $B_{2}$, then $B_{1} \preccurlyeq^{P} B_{2}$.

(b) If $B_{2}$ contains an $\succcurlyeq$-upper bound of $B_{1}$, then $B_{1} \preccurlyeq^{U} B_{2}$.

(c) If $B_{1}$ contains an $\succcurlyeq$-lower bound of $B_{2}$, then $B_{1} \preccurlyeq^{D} B_{2}$.

Proof. The proof of Lemma 2.2 is straightforward, and it is omitted here.

In some set optimization problems, suppose that $(Z, \succcurlyeq)$ is the underlying preordered set and one wants to construct an ordering relation on $2^{Z}$. If we use any one of the preordering relations $\succcurlyeq^{P}, \succcurlyeq^{U}$ and $\succcurlyeq^{D}$ defined above, through the extreme cases in Lemma 2.2, one finds that these preordering relations are still too rough. That means that the ordering relation defined by any one of $\succcurlyeq^{P}, \succcurlyeq^{U}$ and $\succcurlyeq^{D}$ among subsets of $Z$ can be determined by the extreme points ( $\succcurlyeq$-upper bound or $\succcurlyeq$-lower bound) in these considered subsets, which ignores the $\succcurlyeq$-relations between all other points in the considered subsets.

Recall that, for a given preordered set $(Z, \succcurlyeq)$ with cluster space $[Z]$. [Z] is a partition of $Z$ corresponding to the equivalent relation $\backsim$ that is induced by the preorder $\succcurlyeq$ on $Z$. So, $[Z] \subseteq 2^{Z}$. As the preorders $\succcurlyeq^{P}, \succcurlyeq^{U}$ and $\succcurlyeq^{U}$ are restricted on $[Z]$, all become partial orders and they are equivalent. We summarize it as a lemma below.

Lemma 2.3. Let $(Z, \succcurlyeq)$ be a preordered set as stated in Lemma 2.1 with $\succcurlyeq$-cluster space $[Z]$. Then, 
(a) $\left([Z], \succcurlyeq^{P}\right),\left([Z], \succcurlyeq^{U}\right)$ and $\left([Z], \succcurlyeq^{D}\right)$ all are partially ordered sets;

(b) $\succcurlyeq^{P}, \succcurlyeq^{U}$ and $\succcurlyeq^{D}$ are equivalent partial orders on $[Z]$.

Proof. The proof of part (a) in this lemma is straightforward, and it is omitted here. Part (b) is proved by the following assertion: for any $\left[z_{1}\right],\left[z_{2}\right] \in[Z]$,

$$
\left[z_{1}\right] \preccurlyeq^{p}\left[z_{2}\right] \text {, or }\left[z_{1}\right] \preccurlyeq^{U}\left[z_{2}\right] \text {, or }\left[z_{1}\right] \preccurlyeq^{D}\left[z_{2}\right] \text { if and only if } z_{1} \preccurlyeq z_{2} \text {. }
$$

This completes the proof.

In case if $(Z, \preccurlyeq)$ is a partially ordered set, that is, $\succcurlyeq$ is a partial order on $Z$, then all $\succcurlyeq$-clusters are singletons. Therefore, $Z=[Z]$ and $\succcurlyeq, \succcurlyeq^{P}, \succcurlyeq^{U}$ and $\succcurlyeq^{D}$ are equivalent.

2.3. The Order-clustered fixed point theorem. Recall that a partially ordered set is said to be chaincomplete, whenever every chain has the least upper bound (unique). If every chain has the greatest lower bound (unique), then this partially ordered set is said to be re-chain-complete. If a partially ordered set is both chain-complete and re-chain-complete, then it is said to be bi-chain-complete.

A preordered set $(Z, \succcurlyeq)$ is said to be $\succcurlyeq$-chain-complete (or chain-complete), whenever every $\succcurlyeq$-chain $\left\{z_{\alpha}\right\}$ in $Z$ has at least one least $\succcurlyeq$-upper bound (it may not unique). The set of the least $\succcurlyeq$-upper bounds of this given chain is an $\succcurlyeq$-cluster in $Z$ and it is denoted by $\vee\left\{z_{\alpha}\right\}$. $(Z, \succcurlyeq)$ is said to be $\succcurlyeq$-re-chain-complete (simply re-chain-complete), if every $\succcurlyeq$-chain $\left\{Z_{\alpha}\right\}$ in $Z$ has at least one greatest $\succcurlyeq$-lower bound (it may not be unique). The set of the greatest $\succcurlyeq$-lower bounds of this given chain is an $\succcurlyeq$-cluster in $Z$ and it is denoted by $\wedge\left\{z_{\alpha}\right\}$. If $(Z, \succcurlyeq)$ is both chain-complete and re-chain-complete, then it is said to be bi-chain-complete.

Hence, a preordered set $(Z, \succcurlyeq)$ is chain-complete (re-chain-complete), whenever, for any chain $\left\{z_{\alpha}\right\}$ in $Z$, one has $\vee\left\{Z_{\alpha}\right\} \neq \emptyset\left(\wedge\left\{Z_{\alpha}\right\} \neq \emptyset\right)$.

Proposition 2.1. Let $(Z, \succcurlyeq)$ be a preordered set with the cluster space [Z]. Then, the following statements are equivalent

(a) $(Z, \succcurlyeq)$ is an $\succcurlyeq$-chain-complete (re-chain-complete, bi-chain-complete) preordered set;

(b) $\left([Z], \succcurlyeq_{P}\right)$ is an $\succcurlyeq^{P}$-chain-complete (re-chain-complete, bi-chain-complete) poset;

(c) $\left([Z], \succcurlyeq_{U}\right)$ is an $\succcurlyeq^{U}$-chain-complete (re-chain-complete, bi-chain-complete) poset;

(d) $\left([Z], \succcurlyeq_{D}\right)$ is an $\succcurlyeq^{D}$-chain-complete (re-chain-complete, bi-chain-complete) poset.

Proof. From part (b) of Lemma 2.2, we only need to prove that $(Z, \succcurlyeq)$ is a chain-complete (re-chaincomplete, bi-chain-complete) preordered set if and only if $\left([Z], \succcurlyeq^{P}\right)$ is an $\succcurlyeq^{P}$-chain-complete (re-chaincomplete, bi-chain-complete) partially ordered set.

$\Longrightarrow$ Assume that $(Z, \succcurlyeq)$ is $\succcurlyeq$-chain-complete. For arbitrary $\succcurlyeq^{P}$-chain $\left\{\left[z_{\alpha}\right]\right\} \subseteq[Z]$, it corresponds to an $\succcurlyeq$-chain $\left\{Z_{\alpha}\right\} \subseteq Z$. Then, $V_{\succcurlyeq}\left\{Z_{\alpha}\right\}$ exists (here $V_{\succcurlyeq}\left\{Z_{\alpha}\right\}$ is the $\succcurlyeq$-cluster of $\succcurlyeq$-least maximal elements of the chain $\left\{Z_{\alpha}\right\}$, which is a subset of $Z$ ). Let $z=V_{\succcurlyeq}\left\{Z_{\alpha}\right\}$. One can check that $[z]=\vee_{\succcurlyeq}\left\{\left[Z_{\alpha}\right]\right\}$ (here $\vee_{\succcurlyeq}\left\{\left[Z_{\alpha}\right]\right\}$ is the $\succcurlyeq^{P}$-cluster of $\succcurlyeq^{P}$-least maximal elements of the chain $\left\{\left[Z_{\alpha}\right]\right\}$, which is a subset of $[Z]$ ). Hence $\left([Z], \succcurlyeq^{P}\right)$ is $\succcurlyeq^{P}$-chain-complete. All other parts of the conclusions of this lemma can be similarly checked.

Recalling part (i) of Lemma 2.1, for a given preordered set $(Z, \succcurlyeq)$, there are three corresponding preordered sets $\left(2^{Z}, \succcurlyeq^{P}\right),\left(2^{Z}, \succcurlyeq^{U}\right)$ and $\left(2^{Z}, \succcurlyeq^{D}\right)$. Proposition 2.1 says that if $(Z, \succcurlyeq)$ is $\succcurlyeq$-chain-complete 
(re-chain-complete, bi-chain-complete), then the cluster space $[Z]$ as a subset of $2^{Z}$ satisfies that $([Z]$, $\left.\succcurlyeq^{P}\right),\left([Z], \succcurlyeq^{U}\right)$ and $\left([Z], \succcurlyeq^{D}\right)$ all are chain-complete (re-chain-complete, bi-chain-complete) of subsets $\left(2^{Z}, \succcurlyeq^{P}\right),\left(2^{Z}, \succcurlyeq^{U}\right)$ and $\left(2^{Z}, \succcurlyeq^{D}\right)$, respectively. It is worthy to note the following.

Remark 2.2. Suppose that $(Z, \succcurlyeq)$ is ether an $\succcurlyeq$-chain-complete preordered set or an $\succcurlyeq$-chain-complete partially ordered set (re-chain-complete, bi-chain-complete). $\left(2^{Z}, \succcurlyeq^{P}\right),\left(2^{Z}, \succcurlyeq^{U}\right)$ and $\left(2^{Z}, \succcurlyeq^{D}\right)$ may not be $\succcurlyeq^{P}$-chain-complete, $\succcurlyeq^{U}$-chain-complete and $\succcurlyeq^{D}$-chain-complete (re-chain-complete, bi-chain-complete), respectively.

We extend the concepts of universally inductive subsets in partially ordered sets to preordered sets. That will be used in the proof of the existence theorems in this section.

A nonempty subset $A$ of a preordered set $(Z, \succcurlyeq)$ is said to be universally inductive in $Z$ if, for any given $\succcurlyeq$-chain $\left\{x_{\alpha}\right\} \subseteq Z$ satisfying that every element $x_{\beta} \in\left\{x_{\alpha}\right\}$ has an $\succcurlyeq$-upper cover in $A$, then this $\succcurlyeq$-chain $\left\{x_{\alpha}\right\}$ has an $\succcurlyeq$-upper bound in $A$. $A$ is said to be re-universally inductive in $Z$, for any given $\succcurlyeq$-chain $\left\{x_{\alpha}\right\} \subseteq Z$ satisfying that every element $x_{\beta} \in\left\{x_{\alpha}\right\}$ has an $\succcurlyeq$-lower bound in $A$, then this $\succcurlyeq$-chain $\left\{x_{\alpha}\right\}$ has an $\succcurlyeq$-lower bound in $A$. $A$ is said to be bi-universally inductive in $Z$ if, $A$ is both universally inductive and re-universally inductive in $Z$. The empty subset $\emptyset$ of a preordered set $(Z, \succcurlyeq)$ is automatically biuniversally inductive in $Z$. Like Proposition 2.1, which is on the chain-complete properties, regarding to the universally, re-universally and bi-universally inductivities, there are some connections between a preordered set and its cluster space.

Proposition 2.2. Let $(Z, \succcurlyeq)$ be a preordered set with the cluster space $[Z]$. Let $A$ be a nonempty subset of $Z$. Then, the following statements are equivalent

(a) $(A, \succcurlyeq)$ is universally (re-universally, bi-universally) inductive in $(Z, \succcurlyeq)$;

(b) $\left([A], \succcurlyeq^{P}\right)$ is universally (re-universally, bi-universally) inductive in $\left([Z], \succcurlyeq^{P}\right)$;

(c) $\left([A], \succcurlyeq^{U}\right)$ is universally (re-universally, bi-universally) inductive in $\left([Z], \succcurlyeq^{U}\right)$;

(d) $\left([A], \succcurlyeq^{D}\right)$ is universally (re-universally, bi-universally) inductive in $\left([Z], \succcurlyeq^{D}\right)$.

Proof. The proof of this proposition is like the proof of Proposition 2.1 and it is straightforward. It is omitted here.

Definition 2.3. In general, let $\left(S_{1}, \succcurlyeq_{1}\right)$ and $\left(S_{2}, \succcurlyeq_{2}\right)$ be preordered sets. $A$ mapping $g: S_{1} \rightarrow S_{2}$ is said to be order increasing (decreasing) with respect to $\succcurlyeq_{1}$ and $\succcurlyeq_{2}$, whenever, for any $s, t \in S_{1}$,

$$
s \preccurlyeq_{1} t \text { implies } g(s) \preccurlyeq_{2} g(t) \quad\left(g(s) \succcurlyeq_{2} g(t)\right) .
$$

Lemma 2.4. Let $(Z, \succcurlyeq)$ be a preordered set with the cluster space $[Z]$. Let $F: Z \rightarrow 2^{Z}$ be a set-valued mapping. Let $z_{1}, z_{2} \in Z$ with $z_{1} \preccurlyeq z_{2}$.

(i) If $F$ is order increasing with respect to $\succcurlyeq$ and $\succcurlyeq^{U}$, then

$$
F\left(z_{1}\right) \neq \emptyset \text { implies } F\left(z_{2}\right) \neq \emptyset \text {. }
$$

(ii) If $F$ is order increasing with respect to $\succcurlyeq_{\text {and }} \succcurlyeq^{D}$, then

$$
F\left(z_{2}\right) \neq \emptyset \text { implies } F\left(z_{1}\right) \neq \emptyset \text {. }
$$

(iii) If $F$ is order increasing with respect to $\succcurlyeq$ and $\succcurlyeq^{P}$, then

$$
F\left(z_{1}\right) \neq \emptyset \text { if and only if } F\left(z_{2}\right) \neq \emptyset \text {. }
$$


Proof. The proof of Lemma 2.4 is straightforward, and it is omitted here.

Now, we prove an existence theorem of order-clustered fixed points for set valued mappings.

Theorem 2.1. Let $(Z, \succcurlyeq)$ be an $\succcurlyeq$-chain-complete preordered set with the cluster space $[Z]$. Let $F: Z \rightarrow$ $2^{Z}$ be a set-valued mapping. Suppose that $F$ satisfies the following conditions:

(i) $F$ is order increasing with respect to $\succcurlyeq$ and $\succcurlyeq^{U}$;

(ii) for every fixed $z \in Z,\left([F(z)], \succcurlyeq^{U}\right)$ is universally inductive in $\left([Z], \succcurlyeq^{U}\right)$;

(iii) there is $z_{1} \in Z$ such that $\left\{z_{1}\right\} \succcurlyeq^{U} F\left(z_{1}\right)$.

Then, $F$ has an $\succcurlyeq$-clustered fixed point. That is, there is $z^{*}$ such that $\left[z^{*}\right] \in\left[F\left(z^{*}\right)\right]$. More precisely, there are $z^{*}, u \in A$ with $u \backsim z^{*}$ such that

$$
u \in F\left(z^{*}\right)
$$

Moreover, we have

(a) $(\mathfrak{F}(F), \succcurlyeq)$ is a nonempty inductive preordered subset of $Z$;

(b) $F$ has an $\succcurlyeq$-maximal clustered fixed point $u_{1}$ with $u_{1} \succcurlyeq z_{1}$.

Proof. Define a subset of $Z$ by

$$
A=\left\{z \in Z:\{z\} \preccurlyeq^{U} F(z)\right\} .
$$

From condition (iii) in this theorem, $z_{1} \in A$. Hence $A \neq \emptyset$. One can see that, $F(z) \neq \emptyset$, for any $z \in A$.

Next, we first show that $(A, \succcurlyeq)$ has the following properties:

$$
\text { for every } z \in A \text { and for every } v \in F(z) \text { satisfying } z \preccurlyeq v \text {, we have } v \in A \text {. }
$$

As a matter of fact, for every $z \in A$, from $\{z\} \preccurlyeq^{U} F(z)$, there is at least one point $v \in F(z)$ such that $z \preccurlyeq v$. Taking arbitrary $v \in F(z)$ satisfying $z \preccurlyeq v$, from condition (i) in this theorem, it follows that $F(z) \preccurlyeq^{U} F(v)$ (Since $F$ is order increasing with respect to $\succcurlyeq$ and $\succcurlyeq^{U}$, from $z \preccurlyeq v, v \in F(z)$. By Lemma 2.4, we have $F(v) \neq \emptyset)$. So, for the given $v \in F(z)$, there is $w \in F(v)$ such that $v \preccurlyeq w$. It implies $\{v\} \preccurlyeq^{U} F(v)$. Hence $v \in A$. It proves (2.5). Then, we show that $(A, \succcurlyeq)$ is inductive. To this end, take an arbitrary $\succcurlyeq$-chain $z_{\alpha} \subseteq A$. Since $(Z, \succcurlyeq)$ is an $\succcurlyeq$-chain-complete preordered set, one finds that $\left\{z_{\alpha}\right\}$ has a least $\succcurlyeq$-upper bound $w \in Z$. It satisfies

$$
z_{\alpha} \preccurlyeq w, \text { for all index } \alpha \text {. }
$$

From condition (i) in this theorem, we get

$$
F\left(z_{\alpha}\right) \preccurlyeq^{U} F(w) \text {, for all index } \alpha .
$$

Since $z_{\alpha} \in A$, for every index $\alpha$, there is $z_{\alpha} \preccurlyeq v_{\alpha}$ such that $z_{\alpha} \preccurlyeq v_{\alpha}$. Then, from (2.7), there is $w_{\alpha} \in f(w)$ such that $v_{\alpha} \preccurlyeq w_{\alpha}$. We obtain

$$
z_{\alpha} \preccurlyeq v_{\alpha} \preccurlyeq w_{\alpha} \text {. for every index } \alpha \text {. }
$$

From Lemma 2.3, one has

$$
\left[z_{\alpha}\right] \preccurlyeq^{U}\left[v_{\alpha}\right] \preccurlyeq^{U}\left[w_{\alpha}\right] \text {. for every index } \alpha .
$$

Notice that, for every index $\alpha,\left[w_{\alpha}\right] \in[F(w)]$, from condition (ii) in this theorem, $\left([F(z)], \succcurlyeq^{U}\right)$ is universally inductive in $\left([Z], \succcurlyeq^{U}\right)$, and $\left\{\left[z_{\alpha}\right]\right\}$ is an $\succcurlyeq^{U}$-chain in $\left([A], \succcurlyeq^{U}\right) \subset\left([Z], \succcurlyeq^{U}\right)$. Then, there is an element $\left[w^{\prime}\right]$ in $F(w)$ with $w^{\prime} \in f(w)$, such that

$$
\left[z_{\alpha}\right] \preccurlyeq^{U}\left[w^{\prime}\right] \text {, for every index } \alpha \text {. }
$$


It follows from (2.9) that

$$
z_{\alpha} \preccurlyeq w^{\prime} \text {, for every index } \alpha \text {. }
$$

Since $w \in Z$ is a least $\succcurlyeq$-upper bound of $\left\{z_{\alpha}\right\}$, one concludes from (2.10) that

$$
w \preccurlyeq w^{\prime} \in F(w) .
$$

Hence $w \in A$. It implies that $(A, \succcurlyeq)$ is $\succcurlyeq$-chain complete. So, $(A, \succcurlyeq)$ is an inductive preordered set. Taking an arbitrary $\succcurlyeq$-maximal element $z^{*}$ in $(A, \succcurlyeq)$, one sees that there is $u \in F\left(z^{*}\right)$ such that $z^{*} \preccurlyeq u$. From (2.5), one has $u \in A$. Since $z^{*}$ is an $\succcurlyeq$-maximal element $z^{*}$ in $(A, \succcurlyeq)$, from $z^{*} \preccurlyeq u$, it yields that $z^{*} \sim u$, that is, $\left[z^{*}\right]=[u]$. From $u \in F\left(z^{*}\right)$ and Definition 2.2, it implies that $z^{*}$ is an $\succcurlyeq$-clustered fixed point of $F$. Summarizing the above proof about the set $A$ defined in (2.4), we obtain that $(A, \succcurlyeq)$ is an inductive preordered set and every $\succcurlyeq$-maximal element contained in $A$ is an $\succcurlyeq$-clustered fixed point of $F$. Since $\mathfrak{F}(F) \subseteq A$ for arbitrary $\succcurlyeq$-chain $\left\{Z_{\lambda}\right\} \subseteq \mathfrak{F}(F) \subseteq A$, from the above proof, there is an $\succcurlyeq$-maximal element $u$ in $(A, \succcurlyeq)$, which is an $\succcurlyeq$-upper bound of $\left\{z_{\lambda}\right\}$. Meanwhile, $u$ is an $\succcurlyeq$-clustered fixed point of $F$. It shows that $(\mathfrak{F}(F), \succcurlyeq)$ is inductive. For the given point $z_{1}$ in condition (iii) in this theorem, let $Z_{1}=\left\{z \in Z: z \succcurlyeq z_{1}\right\}$. Consider the restriction of $F$ on $Z_{1}$, which is denoted by $F_{1} . F_{1}$ satisfies all conditions (i)-(iii) in this theorem. From the above proof, $(\mathfrak{F}(F), \succcurlyeq)$ is a nonempty inductive of $Z_{1}$. It implies that $F_{1}$ has an $\succcurlyeq$-maximal clustered fixed point. It is clear that $\mathfrak{F}\left(F_{1}\right) \subseteq \mathfrak{F}(F)$ and all $\succcurlyeq$-maximal clustered fixed points of $F_{1}$ are $\succcurlyeq$-maximal clustered fixed points of $F$. It proves (b).

If $(Z, \succcurlyeq)$ is a partially ordered set, then, as a consequence of Theorem 2.1, we obtain the following fixed point theorem immediately which was proved in [14].

Corollary 2.1. Let $(Z, \succcurlyeq)$ be a chain-complete partially ordered set. Let $F: Z \rightarrow 2^{Z}$ be a set-valued mapping. Suppose that $F$ satisfies the following conditions:

(i) $F$ is order increasing with respect to $\succcurlyeq$ and $\succcurlyeq^{U}$;

(ii) for every fixed $z \in Z,(F(z)$, $)$ is universally inductive in $(Z, \succcurlyeq)$;

(iii) there is $z_{1} \in Z$ such that $\left\{z_{1}\right\} \preccurlyeq^{U} F\left(z_{1}\right)$.

Then, $F$ has a fixed point. Moreover, we have

(a) $(\mathscr{F}(F), \succcurlyeq)$ is a nonempty inductive subset of $Z$;

(b) $F$ has an $\succcurlyeq$-maximal fixed point $u_{1}$ with $u_{1} \succcurlyeq z_{1}$.

2.4. Some consequences of the order-clustered fixed point theorem. If we consider the opposite order direction in Theorem 2.1, then we have the following.

Theorem 2.2. Let $(Z, \succcurlyeq)$ be a re-chain-complete preordered set with cluster space $[Z]$. Let $F: Z \rightarrow 2^{Z}$ be a set-valued mapping. Suppose that $F$ satisfies the following conditions:

(i) $F$ is order increasing with respect to $\succcurlyeq$ and $\succcurlyeq^{D}$;

(ii) for every fixed $z \in Z,\left([F(z)], \succcurlyeq^{D}\right)$ is re-universally inductive in $\left([Z], \succcurlyeq^{D}\right)$;

(iii) there is $z_{2} \in$ Zsuch that $\left\{z_{2}\right\} \succcurlyeq^{D} F\left(z_{2}\right)$.

Then, $F$ has an $\succcurlyeq$-clustered fixed point. Moreover, we have

(a) $(\mathfrak{F}(F), \succcurlyeq)$ is a nonempty re-inductive preordered subset of $Z$;

(b) $F$ has an $\succcurlyeq$-minimal clustered fixed point $u_{2}$ with $u_{2} \preccurlyeq z_{2}$. 
Proof. Define a subset of $Z$ by

$$
A^{\prime}=\left\{z \in Z:\{z\} \succcurlyeq^{D} F(z)\right\} .
$$

Then, by using the set $A^{\prime}$, and the proof of Theorem 2.1, we obtain Theorem 2.2 immediately.

By combining Theorems 2.1 and 2.2, we have the following results.

Theorem 2.3. Let $(Z, \succcurlyeq)$ be a bi-chain-complete preordered set with cluster space $[Z]$. Let $F: Z \rightarrow 2^{Z}$ be a set-valued mapping. Suppose that $F$ satisfies the following conditions:

(i) $F$ is order increasing with respect to $\succcurlyeq$ and $\succcurlyeq^{P}$;

(ii) for every fixed $z \in Z,([F(z)]), \succcurlyeq P$ is bi-universally inductive in $\left([Z], \succcurlyeq^{P}\right)$;

(iii) there are $z_{1}, z_{2} \in Z$ such that $\left\{z_{1}\right\} \preccurlyeq^{U} F\left(z_{1}\right)$ and $\left\{z_{2}\right\} \succcurlyeq^{D} F\left(z_{2}\right)$.

Then, $F$ has an $\succcurlyeq$-clustered fixed point. Moreover, we have

(a) $(\mathfrak{F}(F), \succcurlyeq)$ is a nonempty bi-inductive preordered subset of $Z$;

(b) $F$ has an $\succcurlyeq$-maximal clustered fixed point $u_{1}$ with $u_{1} \succcurlyeq z_{1}$;

(c) $F$ has an $\succcurlyeq$-minimal clustered fixed point $u_{2}$ with $u_{2} \preccurlyeq z_{2}$.

If the considered space is a partially ordered set in Theorem 2.3, then we obtain some fixed point theorems on partially ordered sets proved by Fujimoto [2], and Li [13, 14].

Corollary 2.2. Let $(Z, \succcurlyeq)$ be a bi-chain-complete partially ordered set. Let $F: Z \rightarrow 2^{Z}$ be a set-valued mapping. Suppose that $F$ satisfies the following conditions:

(i) $F$ is order increasing with respect to $\succcurlyeq$ and $\succcurlyeq^{P}$;

(ii) for every fixed $z \in Z,(F(z), \succcurlyeq)$ is bi-universally inductive in $(Z, \succcurlyeq)$;

(iii) there are $z_{1}, z_{2} \in Z$ such that $\left\{z_{1}\right\} \preccurlyeq^{U} F\left(z_{1}\right)$ and $\left\{z_{2}\right\} \succcurlyeq^{D} F\left(z_{2}\right)$.

Then, $F$ has a fixed point. Moreover, we have

(a) $(\mathscr{F}(F), \succcurlyeq)$ is a nonempty bi-inductive subset of $Z$;

(b) $F$ has an $\succcurlyeq$-maximal fixed point $u_{1}$ with $u_{1} \succcurlyeq z_{1}$;

(c) $F$ has an $\succcurlyeq$-minimal fixed point $u_{2}$ with $u_{2} \preccurlyeq z_{2}$.

For example, if the underlying space is a complete lattice and the considered mapping is single- valued, then we obtain the Tarskis fixed point theorem immediately; see Abian- Brown [1], Tarski [15] and the references therein.

Corollary 2.3. Let $(Z, \succcurlyeq)$ be a nonempty complete lattice. Let $f: Z \rightarrow Z$ be a single-valued $\succcurlyeq$-increasing mapping. Then, $(\mathscr{F}(f), \succcurlyeq)$ is a nonempty inductive partially ordered set and $f$ has both $\succcurlyeq$-maximal and $\succcurlyeq$-minimal fixed points.

\section{EXTENDED NASH EQUILIBRIUMS AND ORDER-CLUSTERED EXTENDED NASH EQUILIBRIUMS}

\subsection{Definitions of extended Nash equilibriums and order-clustered extended Nash equilibriums.}

In this subsection, by using the concepts of power preorder, upward power preorder and downward power preorder relations defined on the power set of the underlying ordered set, we first generalize the concept of the Nash equilibrium from single-valued mappings to set-valued mappings, which are called the extended Nash equilibrium and order-clustered extended Nash equilibrium. Then, we apply the order-clustered fixed point theorems and fixed point theorems proved in Section 2 to prove the existence 
of the extended Nash equilibrium and order-clustered extended Nash equilibrium for some set-valued mappings in preordered sets.

Let $\left(X, \succcurlyeq_{X}\right)$ and $\left(Y, \succcurlyeq_{Y}\right)$ be preordered spaces. Let $\left(Z, \succcurlyeq_{Z}\right)$ be a preordered space with power, upward power and downward power preorders $\succcurlyeq^{P} \succcurlyeq^{U}$ and $\succcurlyeq^{D}$ on $2^{Z}$, respectively. Denote by $\succcurlyeq_{X Y}$ for the component-wise ordering on $X \times Y$ of $\succcurlyeq_{X}$ and $\succcurlyeq_{Y}$. That is, for $\left(x_{1}, y_{1}\right),\left(x_{2}, y_{2}\right) \in X \times Y$, we say that

$$
\left(x_{2}, y_{2}\right) \succcurlyeq_{X Y}\left(x_{1}, y_{1}\right) \quad \text { if and only if } \quad x_{2} \succcurlyeq_{X} x_{1} \text { and } y_{2} \succcurlyeq_{Y} y_{1} \text {. }
$$

Then, $\succcurlyeq_{X Y}$ is a preorder on $X \times Y$, and $\left(X \times Y, \succcurlyeq_{X Y}\right)$ is a preorder set. Let $C \subseteq X$ and $D \subseteq Y$ be nonempty subsets and let $T: C \times D \rightarrow 2^{Z}$ be a set-valued mapping.

Definition 3.1. A point $\left(x_{0}, y_{0}\right) \in C \times D$ is called an extended Nash equilibrium for a set-valued mapping $T: C \times D \rightarrow 2^{Z}$, if the following order inequalities hold:

$$
T\left(x, y_{0}\right) \preccurlyeq^{U} T\left(x_{0}, y_{0}\right) \preccurlyeq^{D} T\left(x_{0}, y\right), \text { for all }(x, y) \in C \times D .
$$

The set of all extended Nash equilibriums defined by (3.2) of this set-valued mapping $T$ is denoted by $E(T)$. Notice that we can consider the following stronger extended Nash equilibriums:

$$
T\left(x, y_{0}\right) \preccurlyeq^{P} T\left(x_{0}, y_{0}\right) \preccurlyeq^{P} T\left(x_{0}, y\right) \text {, for all }(x, y) \in C \times D .
$$

Like the extension of fixed points on partially ordered spaces to order-clustered fixed points on preordered sets, we generalize the concept of the extended Nash equilibrium to the order-clustered extended Nash equilibrium for set-valued mappings on preordered sets.

Definition 3.2. A point $\left(x_{0}, y_{0}\right) \in C \times D$ is called an $\succcurlyeq_{X Y}$-clustered extended Nash equilibrium for a set-valued mapping $T: C \times D \rightarrow 2^{Z}$ if there is $\left(x_{1}, y_{1}\right) \in C \times D$ with $\left(x_{0}, y_{0}\right) \sim_{X Y}\left(x_{1}, y_{1}\right)$ such that

$$
T\left(x, y_{0}\right) \preccurlyeq^{U} T\left(x_{1}, y_{0}\right) \text { and } T\left(x_{0}, y_{1}\right) \preccurlyeq^{D} T\left(x_{0}, y\right) \text {, for all }(x, y) \in C \times D .
$$

The set of all order-clustered extended Nash equilibriums defined by (3.4) of this set-valued mapping $T$ is denoted by $\mathscr{E}(T)$. Like (3.3), one can study the stronger $\succcurlyeq_{X Y}$-clustered extended Nash equilibrium of the following form

$$
T\left(x, y_{0}\right) \preccurlyeq^{P} T\left(x_{1}, y_{0}\right) \text { and } T\left(x_{0}, y_{1}\right) \preccurlyeq^{P} T\left(x_{0}, y\right) \text {, for all }(x, y) \in C \times D .
$$

The following remark presents the connections between extended Nash equilibriums and order-clustered extended Nash equilibriums.

Remark 3.1. For any set-valued mapping, we have that

(a) every extended Nash equilibrium is an order-clustered extended Nash equilibrium. That is, for any set-valued mapping $T$, one has

$$
E(T) \subseteq \mathscr{E}(T),
$$

(b) if the underlying spaces are partially ordered spaces, then the set of order-clustered extended Nash equilibriums coincides to the set of extended Nash equilibriums.

Considering some strategic games with incomplete preferences, sometimes, on the order equivalent possible strategies, the utilities may be order equivalent. Under this motivation, we introduce the following definition. 
Definition 3.3. A set-valued mapping $T: C \times D \rightarrow 2^{Z}$ is said to be $\succcurlyeq_{X Y}$-cluster invariant if, for any points $\left(x_{0}, y_{0}\right),\left(x_{1}, y_{1}\right) \in C \times D$,

$$
\left(x_{0}, y_{0}\right) \sim_{X Y}\left(x_{1}, y_{1}\right) \text { implies } T\left(x_{0}, y_{0}\right) \sim^{P} T\left(x_{1}, y_{1}\right) .
$$

$T: C \times D \rightarrow 2^{Z}$ is said to be strongly $\succcurlyeq_{X Y}$-cluster invariant if, for any points $\left(x_{0}, y_{0}\right),\left(x_{1}, y_{1}\right) \in C \times D$,

$$
\left(x_{0}, y_{0}\right) \sim_{X Y}\left(x_{1}, y_{1}\right) \text { implies } T\left(x_{0}, y_{0}\right)=T\left(x_{1}, y_{1}\right) .
$$

It is clear to see that the strongly $\succcurlyeq_{X Y}$-cluster invariant property implies the $\succcurlyeq_{X Y}$-cluster invariant property. The $\succcurlyeq_{X Y}$-cluster invariant property provides some connections between extended Nash equilibriums and order-clustered extended Nash equilibriums.

Lemma 3.1. If $T: C \times D \rightarrow 2^{Z}$ is $\succcurlyeq_{X Y}$-cluster invariant, then the set of order-clustered extended Nash equilibriums of $T$ coincides to the set of extended Nash equilibriums; that is,

$$
\mathfrak{F}(T)=\mathscr{F}(T) \text {, if } T \text { is } \succcurlyeq_{X Y} \text {-cluster invariant. }
$$

Proof. Since, for any set-valued mapping $T: C \times D \rightarrow 2^{Z}$, the inclusion $\mathscr{F}(T) \subseteq \mathfrak{F}(T)$ always holds. So, we only need to show that, for this $\succcurlyeq_{X Y}$-cluster invariant $T$, the inclusion $\mathfrak{F}(T) \subseteq \mathscr{F}(T)$ holds. To this end, suppose that $\left(x_{0}, y_{0}\right) \in C \times D$ is an arbitrary $\succcurlyeq_{X Y}$-clustered extended Nash equilibrium for this set-valued mapping $T: C \times D \rightarrow 2^{Z}$. Then, there is $\left(x_{1}, y_{1}\right) \in C \times D$ with $\left(x_{0}, y_{0}\right) \sim_{X Y}\left(x_{1}, y_{1}\right)$ such that

$$
T\left(x, y_{0}\right) \preccurlyeq^{U} T\left(x_{1}, y_{0}\right) \quad \text { and } \quad T\left(x_{0}, y_{1}\right) \preccurlyeq^{D} T\left(x_{0}, y\right) \text {, for all }(x, y) \in C \times D .
$$

From $\left(x_{0}, y_{0}\right) \sim_{X Y}\left(x_{1}, y_{1}\right)$, by the transitive of $\sim_{X Y}$, one gets $\left(x_{1}, y_{0}\right) \sim_{X Y}\left(x_{0}, y_{1}\right) \sim_{X Y}\left(x_{0}, y_{1}\right)$. Since $T: C \times D \rightarrow 2^{Z}$ is $\succcurlyeq_{X Y}$-invariant, it implies that $T\left(x_{1}, y_{0}\right) \sim^{P} T\left(x_{0}, y_{1}\right) \sim^{P} T\left(x_{0}, y_{0}\right)$. Substituting it into (3.8) yields

$$
T\left(x, y_{0}\right) \preccurlyeq^{U} T\left(x_{0}, y_{0}\right) \preccurlyeq^{D} T\left(x_{0}, y\right) \text {, for all }(x, y) \in C \times D .
$$

It implies that $\left(x_{0}, y_{0}\right) \in \mathscr{F}(T)$.

3.2. Existence of extended Nash equilibriums and order-clustered extended Nash equilibriums. Let $\left(X, \succcurlyeq_{X}\right)$ and $\left(Y, \succcurlyeq_{Y}\right)$ be preordered sets. Let $(Z, \succcurlyeq$ be a preordered set with power, upward power and downward power preorders $\succcurlyeq^{P}, \succcurlyeq^{U}$ and $\succcurlyeq^{D}$ on $2^{Z}$, respectively. Let $C \subseteq X$ and $D \subseteq Y$ be nonempty subsets and let $T: C \times D \rightarrow 2^{Z}$ be a set-valued mapping. With respect to the mapping $T$, we define two order optimization set-valued mappings $\Phi: C \rightarrow 2^{D}$ and $\Psi: D \rightarrow 2^{C}$ as follows:

$$
\Phi(s)=\left\{t \in D: T(s, t) \text { is an } \succcurlyeq^{D} \text {-lower bound of }\{T(s, y): y \in D\}\right\} \text {, for any } s \in C
$$

and

$$
\Psi(t)=\left\{s \in C: T(s, t) \text { is an } \succcurlyeq^{U} \text {-upper bound of }\{T(x, t): x \in C\}\right\} \text {, for any } t \in D .
$$

Regarding to the order monotonic of set-valued mappings, we have the following definitions, which will be used in the proof of the main existence theorem in this paper.

Definition 3.4. Let $T: C \times D \rightarrow 2^{Z}$ be a set-valued mapping.

(a) For every $x \in C, T(x, \cdot): D \rightarrow 2^{Z}$ is said to be increasing with respect to $\succcurlyeq_{Y}$ and $\succcurlyeq^{U}$, if, for any $t_{1}, t_{2} \in D, t_{1} \preccurlyeq_{Y} t_{2}$ implies that $T\left(x, t_{1}\right) \preccurlyeq^{U} T\left(x, t_{2}\right)$.

(b) For every $y \in D, T(\cdot, y): C \rightarrow 2^{Z}$ is said to be decreasing with respect to $\succcurlyeq_{X}$ and $\succcurlyeq^{D}$, if, for any $s_{1}, s_{2} \in C, s_{1} \preccurlyeq X s_{2}$ implies that $T\left(s_{1}, y\right) \succcurlyeq^{D} T\left(s_{2}, y\right)$. 
Corresponding to the semi-continuity of real valued functions in real analysis, we introduce the concepts of the order-upper consistent and the order-lower consistent.

Definition 3.5. Let $T: C \times D \rightarrow 2^{Z}$ be a set-valued mapping.

(a) $T$ is said to be $\succcurlyeq^{U}$-upper consistent on $C$, whenever, for any $t_{1}, t_{2} \in D$, if

$$
T\left(x, t_{1}\right) \preccurlyeq^{U} T\left(x, t_{2}\right) \text {, for every } x \in C
$$

then, $\Psi\left(t_{1}\right) \subseteq \Psi\left(t_{2}\right)$. That is, for some $s \in C$, if $T\left(s, t_{1}\right)$ is an $\succcurlyeq^{U}$-upper bound of $\left\{T\left(x, t_{1}\right): x \in C\right\}$, then $T\left(s, t_{2}\right)$ is an $\succcurlyeq^{U}$-upper bound of $\left\{T\left(x, t_{2}\right): x \in C\right\}$.

(b) $T$ is said to be $\succcurlyeq^{D}$-lower consistent on $D$, whenever, for any $s_{1}, s_{2} \in C$, if

$$
T\left(s_{1}, y\right) \succcurlyeq^{D} T\left(s_{2}, y\right) \text {, for every } y \in D
$$

then, $\Phi\left(s_{1}\right) \subseteq \Phi\left(s_{2}\right)$. That is, for some $t \in D$, if $T\left(s_{1}, t\right)$ is an $\succcurlyeq^{D}$-lower bound of $\left\{T\left(s_{1}, y\right): y \in D\right\}$, then $T\left(s_{2}, t\right)$ is an $\succcurlyeq^{D}$-lower bound of $\left\{T\left(s_{2}, y\right): y \in D\right\}$.

Now, we prove an existence theorem of order-clustered extended Nash equilibriums for set-valued mappings on preordered sets.

Theorem 3.1. Let $\left(X, \succcurlyeq_{X}\right)$ and $\left(Y, \succcurlyeq_{Y}\right)$ be preordered sets. Let $(Z, \succcurlyeq)$ be a preordered set with power, upward power and downward power preorders $\succcurlyeq^{P}, \succcurlyeq^{U}$ and $\succcurlyeq^{D}$ on $2^{Z}$, respectively. Let $C \subseteq X$ and $D \subseteq Y$ be nonempty chain-complete subsets and let $T: C \times D \rightarrow 2^{Z}$ be a set-valued mapping. Suppose that $T$ satisfies the following conditions:

(i) for everyx $\in C, T(x, \cdot): D \rightarrow 2^{Z} \backslash\{\emptyset\}$ is increasing on $D$ with respect to $\succcurlyeq_{Y}$ and $\succcurlyeq^{U}$;

(ii) for every $y \in D, T(\cdot, y): C \rightarrow 2^{Z} \backslash\{\emptyset\}$ is decreasing on $C$ with respect to $\succcurlyeq_{X}$ and $\succcurlyeq^{D}$;

(iii) $T$ is $\succcurlyeq^{U}$-upper consistent on $C$ and $\succcurlyeq^{D}$-lower consistent on $D$;

(iv) for every $s \in C,(\Phi(s), \succcurlyeq Y)$ is university inductive in $(D, \succcurlyeq Y)$;

(v) for every $t \in D,\left(\Psi(t), \succcurlyeq_{X}\right)$ is university inductive in $\left(C, \succcurlyeq_{X}\right)$;

(vi) there are $\left(s_{0}, t_{0}\right),\left(s_{1}, t_{1}\right) \in C \times D$ with $t_{1} \in \Phi\left(s_{0}\right)$ and $s_{1} \in \Psi\left(t_{0}\right)$ such that

$$
s_{0} \preccurlyeq X s_{1} \text { and } t_{0} \preccurlyeq Y t_{1} \text {. }
$$

Then, $T$ has an $\succcurlyeq_{X Y}$-clustered extended Nash equilibrium. That is, there are $\left(x_{0}, y_{0}\right),\left(x_{1}, y_{1}\right) \in C \times D$ with $\left(x_{0}, y_{0}\right) \sim_{X Y}\left(x_{1}, y_{1}\right)$ such that

$$
T\left(x, y_{0}\right) \preccurlyeq^{U} T\left(x_{1}, y_{0}\right) \text { and } T\left(x_{0}, y_{1}\right) \preccurlyeq^{D} T\left(x_{0}, y\right), \text { for all }(x, y) \in C \times D .
$$

Moreover, one has

(a) $\left(\mathscr{E}(T), \succcurlyeq_{X Y}\right)$ is a nonempty inductive subset in $\left(C \times D, \succcurlyeq_{X Y}\right)$;

(b) $T$ has an $\succcurlyeq_{X Y}$-maximal clustered extended Nash equilibrium $\left(x_{0}, y_{0}\right) \succcurlyeq_{X Y}\left(s_{0}, t_{0}\right)$.

Proof. With the component-wise ordering $\succcurlyeq_{X Y}$ of $\succcurlyeq_{X}$ and $\succcurlyeq_{Y}$ on $X \times Y,\left(C \times D, \succcurlyeq_{X Y}\right)$ is a preorder subset of $X \times Y$. The power, upward power and downward power preorders on $2^{X \times Y}$ are denoted by $\succcurlyeq_{X Y}^{P}, \succcurlyeq_{X Y}^{U}$, and $\succcurlyeq_{X Y}^{D}$, respectively. Define a mapping $F: C \times D \rightarrow 2^{C \times D}$ by

$$
F(s, t)=\Psi(t) \times \Phi(s), \text { for }(s, t) \in C \times D .
$$


For any $\left(s_{1}, t_{1}\right),\left(s_{2}, t_{2}\right) \in C \times D$ with $\left(s_{2}, t_{2}\right) \succcurlyeq_{X Y}\left(s_{1}, t_{1}\right)$, we have $s_{2} \succcurlyeq_{X} s_{1}$ and $t_{2} \succcurlyeq_{Y} t_{1}$. For these given points $s_{1}, s_{2} \in C$, we recall that

$$
\Phi\left(s_{1}\right)=\left\{t \in D: T\left(s_{1}, t\right) \text { is an } \succcurlyeq^{D} \text {-lower bound of }\left\{T\left(s_{1}, y\right): y \in D\right\}\right\},
$$

and

$$
\Phi\left(s_{2}\right)=\left\{t \in D: T\left(s_{2}, t\right) \text { is an } \succcurlyeq^{D} \text {-lower bound of }\left\{T\left(s_{2}, y\right): y \in D\right\}\right\} .
$$

From $s_{1} \preccurlyeq X s_{2}$, by the decreasing part in condition (ii) in this theorem, it implies that

$$
T\left(s_{1}, y\right) \succcurlyeq^{D} T\left(s_{2}, y\right) \text {, for every } y \in D \text {. }
$$

Since $T$ is $\succcurlyeq^{D}$-lower consistent on $D$, one finds from condition (iii) and (3.10) that

$$
\Phi\left(s_{1}\right) \subseteq \Phi\left(s_{2}\right), \text { for } s_{1} \preccurlyeq X s_{2} .
$$

For $t_{1}, t_{2} \in D$, we have

$$
\Psi\left(t_{1}\right)=\left\{s \in C: T\left(s, t_{1}\right) \text { is an } \succcurlyeq^{U} \text {-upper bound of }\left\{T\left(x, t_{1}\right): x \in C\right\}\right\},
$$

and

$$
\Psi\left(t_{2}\right)=\left\{s \in C: T\left(s, t_{2}\right) \text { is an } \succcurlyeq^{U} \text {-upper bound of }\left\{T\left(x, t_{2}\right): x \in C\right\}\right\} .
$$

From $t_{1} \preccurlyeq_{Y} t_{2}$, by the increasing property of $T$ in condition (i) in this theorem, it implies that

$$
T\left(x, t_{1}\right) \preccurlyeq^{U} T\left(x, t_{2}\right) \text {, for any fixed } x \in C .
$$

From condition (iii), $T$ is $\succcurlyeq^{U}$-upper consistent on $D$. By (3.12), we obtain

$$
\Psi\left(t_{1}\right) \subseteq \Psi\left(t_{2}\right), \text { for } t_{1} \preccurlyeq t_{2} .
$$

From (3.11) and (3.13), we obtain that, for any $\left(s_{1}, t_{1}\right),\left(s_{2}, t_{2}\right) \in C \times D$, if $\left(s_{2}, t_{2}\right) \succcurlyeq_{X Y}\left(s_{1}, t_{1}\right)$,

$$
\Phi\left(s_{1}\right) \times \Psi\left(t_{1}\right) \subseteq \Phi\left(s_{2}\right) \times \Psi\left(t_{2}\right)
$$

From (3.9), it follows that, for any $\left(s_{1}, t_{1}\right),\left(s_{2}, t_{2}\right) \in C \times D$, if $\left(s_{2}, t_{2}\right) \succcurlyeq_{X Y}\left(s_{1}, t_{1}\right)$,

$$
F\left(s_{1}, t_{1}\right) \subseteq F\left(s_{2}, t_{2}\right) .
$$

It implies

$$
F\left(s_{1}, t_{1}\right) \preccurlyeq_{X Y}^{U} F\left(s_{2}, t_{2}\right) .
$$

Hence $F: C \times D \rightarrow 2^{C \times D}$ is increasing with respect to the orders $\succcurlyeq_{X Y}$ and $\succcurlyeq_{X Y}^{U}$. Hence $F$ satisfies condition (i) in Theorem 2.1. From conditions (iv)-(v) in this theorem, for every given $(s, t) \in C \times$ $D,(\Phi(s), \succcurlyeq Y)$ is university inductive in $(D, \succcurlyeq r)$ and $\left(\Psi(t), \succcurlyeq_{X}\right)$ is university inductive in $(C, \succcurlyeq X)$. It implies that, for every $(s, t) \in C \times D,\left(F(s, t), \succcurlyeq_{X Y}\right)$ is university inductive in $\left(C \times D, \succcurlyeq_{X Y}\right)$. Hence $F$ satisfies condition (ii) in Theorem 2.1. For the given points $\left(s_{0}, t_{0}\right),\left(s_{1}, t_{1}\right) \in C \times D$ in this theorem with $t_{1} \in \Phi\left(s_{0}\right)$ and $s_{1} \in \Psi\left(t_{0}\right)$, we have $\left(s_{1}, t_{1}\right) \in F\left(s_{0}, t_{0}\right)$. From $s_{1} \succcurlyeq_{X} s_{0}$ and $t_{1} \succcurlyeq_{Y} t_{0}$, we obtain

$$
\left\{\left(s_{0}, t_{0}\right)\right\} \preccurlyeq_{X Y}^{U} F\left(s_{0}, t_{0}\right) .
$$

By (3.16), $F: C \times D \rightarrow 2^{C \times D}$ satisfies condition (iii) in Theorem 2.1. Hence, $F$ has an $\succcurlyeq_{X Y}$-clustered fixed point $\left(x_{0}, y_{0}\right)$. So, there exits $\left(x_{1}, y_{1}\right) \in C \times D$ with $\left(x_{0}, y_{0}\right) \sim_{X Y}\left(x_{1}, y_{1}\right)$ such that

$$
\left(x_{1}, y_{1}\right) \in F\left(x_{0}, y_{0}\right)=\Psi\left(y_{0}\right) \times \Phi\left(x_{0}\right) .
$$


It implies that $x_{1} \in \Psi\left(y_{0}\right)$ and $y_{1} \in \Phi\left(x_{0}\right)$. That is,

$$
\begin{aligned}
& x_{1} \in \Psi\left(y_{0}\right)=\left\{s \in C: T\left(s, y_{0}\right) \text { is an } \succcurlyeq^{U} \text {-upper bound of }\left\{T\left(x, y_{0}\right): x \in C\right\}\right\}, \\
& y_{1} \in \Phi\left(x_{0}\right)=\left\{t \in D: T\left(x_{0}, t\right) \text { is an } \succcurlyeq^{D} \text {-lower bound of }\left\{T\left(x_{0}, y\right): y \in D\right\}\right\} .
\end{aligned}
$$

It follows that

$$
T\left(x_{1}, y_{0}\right) \text { is an } \succcurlyeq^{U} \text {-upper bound of }\left\{T\left(x, y_{0}\right): x \in C\right\}
$$

and

$$
T\left(x_{0}, y_{1}\right) \text { is an } \succcurlyeq^{D} \text {-lower bound of }\left\{T\left(x_{0}, y\right): y \in D\right\} \text {. }
$$

Thus,

$$
T\left(x, y_{0}\right) \preccurlyeq^{U} T\left(x_{1}, y_{0}\right) \quad \text { and } \quad T\left(x_{0}, y_{1}\right) \preccurlyeq^{D} T\left(x_{0}, y\right), \quad \text { for all }(x, y) \in C \times D .
$$

Hence the point $\left(x_{0}, y_{0}\right)$ is an $\succcurlyeq_{X Y}$-clustered extended Nash equilibrium of mapping $T$. The conclusions (a) and (b) immediately follows from conclusions (a) and (b) of Theorem 2.1.

By Remark 3.1 and Lemma 3.1, we obtain an existence theorem for extended Nash equilibrium for set-valued mappings as a consequence of Theorem 3.1.

Theorem 3.2. Let $\left(X, \succcurlyeq_{X}\right)$ and $\left(Y, \succcurlyeq_{Y}\right)$ be preordered spaces and let $\left(Z, \succcurlyeq_{)}\right)$be a preordered space. Let $C \subseteq X$ and $D \subseteq Y$ be nonempty chain-complete subsets and let $T: C \times D \rightarrow 2^{Z}$ be a set-valued mapping. In addition to conditions ( $i$-vi) in Theorem 3.1, if one of the following conditions satisfies:

(vii) $\left(X, \succcurlyeq_{X}\right)$ and $\left(Y, \succcurlyeq_{Y}\right)$ both are partially ordered sets;

(viii) $T: C \times D \rightarrow 2^{Z}$ is $\succcurlyeq_{X Y}$-invariant.

Then, $T$ has an extended Nash equilibrium. That is, there is $\left(x_{0}, y_{0}\right) \in C \times D$ such that

$$
T\left(x, y_{0}\right) \preccurlyeq^{U} T\left(x_{0}, y_{0}\right) \preccurlyeq^{D} T\left(x_{0}, y\right) \text {, for all }(x, y) \in C \times D \text {. }
$$

Moreover, one has

(a) $\left(E(T), \succcurlyeq_{X Y}\right)$ is a nonempty inductive subset in $\left(C \times D, \succcurlyeq_{X Y}\right)$;

(b) $T$ has an $\succcurlyeq_{X Y}$-maximal extended Nash equilibrium $\left(x_{0}, y_{0}\right) \succcurlyeq_{X Y}\left(s_{0}, t_{0}\right)$.

\section{APPLICATIONS TO EXTENDED NASH EQUILIBRIUM PROBLEMS FOR SINGLE VALUED MAPPINGS}

4.1. Existence of extended Nash equilibriums for single valued mappings. Let $(Z, \succcurlyeq)$ be a preordered set with power, upward power and downward power preorders $\succcurlyeq^{P}, \succcurlyeq U$ and $\succcurlyeq^{D}$ on $2^{Z}$, respectively. As we discussed in Lemma 2.1, $\left(2^{Z}, \succcurlyeq^{P}\right),\left(2^{Z}, \succcurlyeq^{U}\right)$ and $\left(2^{Z}, \succcurlyeq^{D}\right)$ all are preorder sets. In general, $\succcurlyeq^{P}, \succcurlyeq^{U}$ and $\succcurlyeq^{D}$ are different preorders on $2^{Z}$. Therefore, $\left(2^{Z}, \succcurlyeq^{P}\right),\left(2^{Z}, \succcurlyeq^{U}\right)$ and $\left(2^{Z}, \succcurlyeq^{D}\right)$ are different preordered sets with the same underlying set $2^{Z}$. Write $\mathscr{Z}=\left\{\{z\} \in 2^{Z}: z \in Z\right\}$. Then, $\mathscr{Z} \subseteq 2^{Z}$.As $\succcurlyeq^{P}, \succcurlyeq^{U}$ and $\succcurlyeq^{D}$ are restricted on $\mathscr{Z}$, which are equivalent to $\succcurlyeq$. We have

$$
\left(\mathscr{Z}, \succcurlyeq^{P}\right)=\left(\mathscr{Z}, \succcurlyeq^{U}\right)=\left(\mathscr{Z}, \succcurlyeq^{D}\right)=(\mathscr{Z}, \succcurlyeq) .
$$

(4.1) provides the crucial connections for the applications of the existence theorems of extended Nash equilibriums for set-valued mappings to extended Nash equilibrium problems for single-valued mappings. First, we discuss the concepts of the upper consistent and the lower consistent, and rewrite optimization functions $\Phi$ and $\Psi$ for single-valued mappings. Let $\left(X, \succcurlyeq_{X}\right),\left(Y, \succcurlyeq_{Y}\right)$ and $\left(Z, \succcurlyeq_{)}\right)$be preordered sets. Let $C \subseteq X$ and $D \subseteq Y$ be nonempty subsets and let $T: C \times D \rightarrow Z$ be a single-valued mapping. With respect 
to the single-valued mapping $T$, we write the two optimization set-valued mappings $\varphi: C \rightarrow 2^{D}$ and $\psi: D \rightarrow 2^{C}$ as follows:

$$
\varphi(s)=\{t \in D: T(s, t) \text { is an } \succcurlyeq-\text { lower bound of }\{T(s, y): y \in D\}\}, \text { for any } s \in C,
$$

and

$$
\psi(t)=\{s \in C: T(s, t) \text { is an } \succcurlyeq \text {-upper bound of }\{T(x, t): x \in C\}\} \text {, for any } t \in D .
$$

From Definition 3.4, the order upper consistent and order lower consistent properties become the following forms for single-valued mappings

(a) $T$ is said to be $\succcurlyeq$-upper consistent on $C$, whenever, for any $t_{1}, t_{2} \in D$, if

$$
T\left(x, t_{1}\right) \preccurlyeq T\left(x, t_{2}\right) \text {, for every } x \in C,
$$

then, $\psi\left(t_{1}\right) \subseteq \psi\left(t_{2}\right)$. That is, for some $s \in C$, if $T\left(s, t_{1}\right)$ is an $\succcurlyeq$-upper bound of $\left\{T\left(x, t_{1}\right): x \in C\right\}$, then $T\left(s, t_{2}\right)$ is an $\succcurlyeq$-upper bound of $\left\{T\left(x, t_{2}\right): x \in C\right\}$;

(b) $T$ is said to be $\succcurlyeq$-lower consistent on $D$, whenever, for any $s_{1}, s_{2} \in C$, if

$$
T\left(s_{1}, y\right) \succcurlyeq T\left(s_{2}, y\right) \text {, for every } y \in D,
$$

then, $\varphi\left(s_{1}\right) \subseteq \varphi\left(s_{2}\right)$. That is, for some $t \in D$, if $T\left(s_{1}, t\right)$ is an $\succcurlyeq-$ lower bound of $\left\{T\left(s_{1}, y\right): y \in D\right\}$, then $T\left(s_{2}, t\right)$ is an $\succcurlyeq$-lower bound of $\left\{T\left(s_{2}, y\right): y \in D\right\}$.

By using the connection (4.1), we have an existence result for order-clustered Nash equilibrium of single valued mappings as a consequence of Theorem 3.1.

Corollary 4.1. Let $\left(X, \succcurlyeq_{X}\right),\left(Y, \succcurlyeq_{Y}\right)$ and $\left(Z, \succcurlyeq_{)}\right)$be preordered sets. Let $C \subseteq X$ and $D \subseteq Y$ be nonempty chain-complete subsets and let $T: C \times D \rightarrow Z$ be a single-valued mapping. Suppose that $T$ satisfies the following conditions:

(i) for every $x \in C, T(x, \cdot): D \rightarrow Z$ is increasing on $D$ with respect to $\succcurlyeq_{Y}$ and $\succcurlyeq_{\text {; }}$

(ii) for every $y \in D, T(\cdot, y): C \rightarrow Z$ is decreasing on $C$ with respect to $\succcurlyeq_{X}$ and $\succcurlyeq_{\text {; }}$;

(iii) $T$ is $\succcurlyeq$-upper consistent on $C$ and $\succcurlyeq$-lower consistent on $D$;

(iv) for every $s \in C,(\varphi(s), \succcurlyeq Y)$ is university inductive in $(D, \succcurlyeq Y)$;

(v) for every $t \in D,\left(\psi(t), \succcurlyeq_{X}\right)$ is university inductive in $\left(C, \succcurlyeq_{X}\right)$;

(vi) there are $\left(s_{0}, t_{0}\right),\left(s_{1}, t_{1}\right) \in C \times D$ with $t_{1} \in \varphi\left(s_{0}\right)$ and $s_{1} \in \psi\left(t_{0}\right)$ such that

$$
s_{0} \preccurlyeq X s_{1} \text { and } t_{0} \preccurlyeq Y t_{1} \text {. }
$$

Then, $T$ has an $\succcurlyeq_{X Y}$-clustered extended Nash equilibrium. That is, there are $\left(x_{0}, y_{0}\right),\left(x_{1}, y_{1}\right) \in C \times D$ with $\left(x_{0}, y_{0}\right) \sim_{X Y}\left(x_{1}, y_{1}\right)$ such that

$$
T\left(x, y_{0}\right) \preccurlyeq T\left(x_{1}, y_{0}\right) \text { and } T\left(x_{0}, y_{1}\right) \preccurlyeq T\left(x_{0}, y\right) \text {, for all }(x, y) \in C \times D \text {. }
$$

Moreover, one has

(a) $\left(\mathscr{E}(T), \succcurlyeq_{X Y}\right)$ is a nonempty inductive subset in $\left(C \times D, \succcurlyeq_{X Y}\right)$;

(b) $T$ has an $\succcurlyeq_{X Y}$-maximal clustered extended Nash equilibrium $\left(x_{0}, y_{0}\right) \succcurlyeq_{X Y}\left(s_{0}, t_{0}\right)$.

Corollary 4.2. Let $\left(X, \succcurlyeq_{X}\right),\left(Y, \succcurlyeq_{Y}\right)$ and $\left(Z, \succcurlyeq_{)}\right.$be preordered sets. Let $C \subseteq X$ and $D \subseteq Y$ be nonempty chain-complete subsets and let $T: C \times D \rightarrow Z$ be a single-valued mapping. In addition to conditions ( $i$-vi) in Corollary 4.1, if $T$ satisfies one of the following two conditions:

(vii) $\left(X, \succcurlyeq_{X}\right)$ and $\left(Y, \succcurlyeq_{Y}\right)$ both are partially ordered sets; 
(viii) $T: C \times D \rightarrow Z$ is $\succcurlyeq_{X Y}$-invariant.

Then, $T$ has an extended Nash equilibrium. That is, there is $\left(x_{0}, y_{0}\right) \in C \times D$ such that

$$
T\left(x, y_{0}\right) \preccurlyeq^{U} T\left(x_{0}, y_{0}\right) \preccurlyeq^{D} T\left(x_{0}, y\right), \text { for all }(x, y) \in C \times D \text {. }
$$

\section{Moreover, one has}

(a) $\left(E(T), \succcurlyeq_{X Y}\right)$ is a nonempty inductive subset in $\left(C \times D, \succcurlyeq_{X Y}\right)$;

(b) $T$ has an $\succcurlyeq_{X Y}$-maximal extended Nash equilibrium $\left(x_{0}, y_{0}\right) \succcurlyeq_{X Y}\left(s_{0}, t_{0}\right)$.

4.2. An example. In this subsection, we provide an example of applications of Corollaries 4.1 and 4.2 to the existence of Nash equilibriums of real functions. One of the reasons for us to list it as a separable subsection is that we will make some different notations to distinct the order-clusters, order-intervals in ordered sets and the regular intervals in the set of real numbers. Meanwhile, we show in details that how to use Corollaries 4.1 and 4.2 to prove the existence of Nash equilibriums of real valued functions.

Example 4.1. Let $m, n$ be positive integers. Let $R^{m}, R^{n}$ denote the Euclidean spaces of dimensions $m, n$, respectively. Let $R$ denote the set of real numbers. Define a function $T: R^{m} \times R^{n} \rightarrow R$ by

$$
T(x, y)=-\sum_{i=1}^{m} x_{i}^{2}+\sum_{j=1}^{n} y_{j}^{2}, \text { for all } x=\left(x_{1}, x_{2}, \ldots, x_{m}\right) \in R^{m}, y=\left(y_{1}, y_{2}, \ldots, y_{n}\right) \in R^{n} .
$$

Then, $T$ has a Nash equilibrium (a saddle point).

Proof. For $k=m, n$, we define an ordering relation $\succsim_{k}$ on $R^{k}$ by the absolute values as follows: for any $r_{1}, r_{2} \in R^{k}$, we say that

$$
r_{1} \precsim_{k} r_{2} \quad \text { if and only if } \quad\left|r_{1}\right| \leq\left|r_{2}\right| .
$$

One can check that $\succsim_{k}$ is a preorder on $R^{k}$. So, for $k=m, n,\left(R^{k}, \succsim_{k}\right)$ is a preordered set (notice that, since $\succsim_{k}$ does not have the linearity properties on the vector space $R^{k},\left(R^{k}, \succsim_{k}\right)$ is not a preordered vector space). For any $r_{1}, r_{2} \in\left(R^{k}, \succsim_{k}\right)$ with $r_{1} \precsim_{k} r_{2}$, the $\succsim_{k}$-interval in $\left(R^{k}, \succsim_{k}\right)$ is denoted by

$$
\left[r_{1}, r_{2}\right]_{\succsim k}=\left\{r \in R: r_{1} \precsim_{k} r \precsim_{k} r_{2}\right\} .
$$

For any $q_{1}, q_{2} \in R$ with $q_{1} \leq q_{2}$, the regular interval in $R$ is denoted by the regular notation

$$
\left[q_{1}, q_{2}\right]=\left\{q \in R: q_{1} \leq q \leq q_{2}\right\} .
$$

For $k=m, n$, let $\theta$ denote the origin of $\succsim_{k}$, if there is no confusion caused. It follows that, for any $r \in\left(R^{k}, \succsim_{k}\right)$ with $r \neq \theta$, the $\succsim_{k}$-cluster in $\left(R^{k}, \succsim_{k}\right)$ containing $r$ is the sphere in $R^{k}$ with center $\theta$ and radius $|r|$. That is,

$$
[r]_{\succsim_{k}}=\left\{\left(t_{1}, t_{2}, \ldots, t_{k}\right) \in R^{k}: \sum_{i=1}^{k} t_{i}^{2}=|r|^{2}\right\}
$$

and $q_{1}, q_{2} \in R$. By (4.3) and (4.4), the $\succsim_{k}$-intervals in $\left(R^{k}, \succsim_{k}\right)$ are the regions between spheres that can be represented as follows: for any $r_{1}, r_{2} \in\left(R^{k}, \succsim_{k}\right)$ with $r_{1} \precsim_{k} r_{2}$,

$$
\left[r_{1}, r_{2}\right]_{\succsim k}=\left\{\left(t_{1}, t_{2}, \ldots, t_{k}\right) \in R^{k}:\left|r_{1}\right|^{2} \leq \sum_{i=1}^{k} t_{i}^{2} \leq\left|r_{2}\right|^{2}\right\} .
$$


For the simplicity in this subsection, let $\succsim_{m+n}$ denote the component-wise ordering of $\succsim_{m}$ and $\succsim_{n}$ on $R^{m} \times R^{n}$ as defined in (3.1). Then, $\left(R^{m} \times R^{n}, \succsim_{m+n}\right)$ is a preorder set. For arbitrary given positive numbers $c, d$, let

$$
C=\left\{\left(x_{1}, x_{2}, \ldots, x_{m}\right) \in R^{m}: \sum_{i=1}^{m} x_{i}^{2} \leq c^{2}\right\} \text { and } D=\left\{\left(y_{1}, y_{2}, \ldots, y_{n}\right) \in R^{n}: \sum_{j=1}^{n} y_{j}^{2} \leq d^{2}\right\} .
$$

One can check that $\left(C \times D, \succsim_{m+n}\right)$ is an $\succsim_{m+n}$-bi-chain complete subset of $\left(R^{m} \times R^{n}, \succsim_{m+n}\right)$. In Corollary 4.1, we take $\left(X, \succcurlyeq_{X}\right)=\left(R^{m}, \succsim_{m}\right),\left(Y, \succcurlyeq_{Y}\right)=\left(R^{n}, \succsim_{n}\right)$ and $(Z, \succcurlyeq)=(R, \geq)$.

Next we check that $T$ satisfies all conditions in Corollary 4.1 on $C \times D$. From the definition of $T$ in (4.2), one sees that, for every $x \in C, T(x, \cdot): D \rightarrow R$ is increasing on $D$ with respect to $\succsim_{m}$ and $\geq$; for every $y \in D, T(\cdot, y): C \rightarrow R$ is decreasing on $C$ with respect to $\succsim_{n}$ and $\geq$. So, $T$ satisfies conditions (i) and (ii) in Corollary 4.1. On can check that, for any $s \in C$,

$$
\varphi(s)=\{t \in D: T(s, t) \text { is an } \geq \text {-lower bound of }\{T(s, y) \in R: y \in D\}\}=\{\theta\}
$$

and, for any $t \in D$

$$
\psi(t)=\{s \in C: T(s, t) \text { is an } \geq \text {-upper bound of }\{T(x, t) \in R: x \in C\}\}=\{\theta\} .
$$

It implies that $T$ satisfies conditions (iii)-(v) in Corollary 4.1. Take $\left(s_{0}, t_{0}\right)=\left(s_{1}, t_{1}\right)=(\theta, \theta)$. From (4.7) and (4.8), we see that condition (iv) is satisfied. It is clear to see that the map $T: C \times D \rightarrow R$ is $\succsim_{m+n}$-invariant. From Corollary 4.2, $T$ has a Nash equilibrium on $C \times D$ (it is clear to see that $(\theta, \theta)$ is the Nash equilibrium of $T$ on $C \times D)$. Since $C \times D$ is arbitrary taken, we can check that $(\theta, \theta)$ is the Nash equilibrium of $T$ on $R^{m} \times R^{n}$.

\section{Acknowledgment}

The authors would like to thank Xiaolong Qin for his great help.

\section{REFERENCES}

[1] S. Abian, A. Brown, A theorem on partially ordered sets with applications to fixed point theorem, Canad. J. Math. 13, (1961) 78-83.

[2] A. Fujimoto, An extension of Tarskis fixed point theorem and its applications to isotone complementarity problems, Math. Program. 28 (1984), 116-118.

[3] A. Göpfert, H. Riahi, C. Tammer, C. Zălinescu, Variational Methods in Partially Ordered Spaces, Springer, New York, (2003)

[4] J. Jahn, Vector Optimization. Theory, Applications and Extensions, Springer, Berlin, (2004)

[5] A.A. Khan, C. Tammer. C. Zălinescu, Set-valued optimization an introduction with applications, Springer-Verlag, Berlin Heidelberg, 2015.

[6] I.V. Konnov, J. C. Yao, On the generalized vector variational inequality problem, J. Math. Anal. Appl. 206 (1997), 42-58.

[7] D. Kuroiwa, The natural criteria in set-valued optimization, RIMS Kokyuroku 1031 (1998), 85-90.

[8] D. Kuroiwa, Lagrange duality of set-valued optimization with natural criteria, RIMS Kokyuroku 1068 (1998), 164-170.

[9] D. Kuroiwa, Some duality theorems of set-valued optimization, RIMS Kokyuroku 1079 (1999), 15-19.

[10] D. Kuroiwa, On natural criteria in set-valued optimization, RIMS Kokyuroku, 1048 (1998), 86-92.

[11] D. Kuroiwa, Some duality theorems of set-valued optimization with natural criteria. In: Tanaka, T. (ed.) Proceedings of the International Conference on Nonlinear Analysis and Convex Analysis, pp. 221-228. World Scientific, Singapore (1999)

[12] D. Kuroiwa, Existence theorems of set optimization with set-valued maps. J. Inf. Optim. Sci. 24 (2003), 73-84. 
[13] J. Li, Several extensions of the AbianCBrown fixed point theorem and their applications to extended and generalized Nash equilibria on chain-complete posets, J. Math. Anal. Appl. 409 (2014), 1084-1092.

[14] J. Li, Inductive properties of fixed point sets of mappings on posets and on partially ordered topological spaces, Fixed Point Theory Appl. 2015 (2015), Article ID 211.

[15] A. Tarski, A lattice-theoretical fixed point theorem and its applications, Pacific J. Math. 5 (1955) 285-309.

[16] L. Xie, J. Li, A. Petrusel, J. C. Yao, Order-clustered fixed point theorems and their applications to Pareto equilibrium problems, Fixed Point Theory, 18 (2017), 755-772.

[17] H. K. Xu, A variable Krasnosel'skii-Mann algorithm and the multiple sets split feasibility problem, Inverse Prob. 22 (2006), 2021-2034.

[18] C. Zhang, Y. Wang, Applications of order-theoretic fixed point theorems to discontinuous quasi-equilibrium problems, Fixed Point Theory Appl. 2015 (2015), Article ID 54. 